\title{
On distances derived from symmetric difference functions
}

\author{
Isabel Aguiló ${ }^{1}$, Tomasa Calvo ${ }^{2}$, Javier Martín ${ }^{1}$, Gaspar Mayor $^{1}$, Jaume Suñer ${ }^{1}$ \\ ${ }^{1}$ University of Balearic Islands, Palma de Mallorca, Spain \\ ${ }^{2}$ University of Alcalá, Alcalá de Henares, Spain
}

\begin{abstract}
Once introduced a definition of symmetric difference function on the unit real interval $[0,1]$, we consider a method to construct such functions based on a triplet formed by a t-norm, a t-conorm and a strong negation. Our main goal is to characterize those triplets that define symmetric difference functions which are distances.
\end{abstract}

Keywords: symmetric difference function, t-norm, t-conorm, strong negation, distance.

\section{Introduction}

Motivated by generalizations of the classical symmetric difference of sets, Alsina introduced in [3] (see also [5]) the idea of constructing distances from a t-norm $T$ and its dual $T^{*}: T^{*}(a, b)=1-T(1-a, 1-b)$. Thus given a tnorm $T$, Alsina defines $d_{T}(a, b)=T^{*}(a, b)-T(a, b)$ if $a \neq b, d_{T}(a, a)=0$, and proves that "if a t-norm $T$ is a copula then $d_{T}$ is a distance". There are examples of continuous non-strict Archimedean t-norms that are not copulas and that generate distances (see [1]), proving that for continuous t-norms the reciprocal of the Alsina's result is not true. In [1] a characterization of those t-norms having zero region $\{(a, b) ; a+b \leq 1\}$ that induce distances is given, however the complete characterization of those t-norms that induce distances is still an open problem. The problem of generating distances from a more general pair $(S, T)$ of a t-conorm and a t-norm is also studied in [1].

In the same way that the linguistic "or" has the functional model given by t-conorms, a functional model for the linguistic "either or" by means of symmetric difference functions can be considered (see [4]). By generalizing the classical expression of set theory "either $A$ or $B^{\prime \prime}=\left(A \cap B^{c}\right) \cup\left(B \cap A^{c}\right)$, we can consider a class of symmetric difference functions of the form $\triangle(a, b)=S(T(a, N(b)), T(b, N(a))$ where $T, S, N$ are a t-norm, a t-conorm and a strong negation respectively. Our main concern in this paper is to give a characterization of those triplets $(T, S, N)$ such that the symmetric difference functions $\triangle$ associated to them are distances.
In Section 2 basic definitions, examples and results are presented. Section 3 contains all the main results of the contribution.

\section{Preliminaries}

We begin with the definitions of t-norm, t-conorm and copula, and some properties and basic examples (see [5] and [7]).

Definition 1 Let us consider functions $T, S:[0,1]^{2} \rightarrow[0,1]$. We say that $T$ is a t-norm if it is increasing in each variable, commutative, associative and has neutral element 1 . We say that $S$ is a t-conorm if it is increasing in each variable, commutative, associative and has neutral element 0 .

Definition 2 A function $N$ from $[0,1]$ onto itself is a strong negation if it is decreasing and involutive $\left(N^{2}=i d\right)$.

Given a strong negation $N$, the $N$-dual t-conorm of a t-norm $T$ is $T^{*}(a, b)=N(T(N(a), N(b)))$. Given a t-norm $T$, a t-conorm $S$, and a strong negation $N$, we say that $(T, S, N)$ is a De Morgan triplet if $T$ and $S$ are $N$-dual.

Example 1 Basic t-norms are the minimum $M(a, b)=\min (a, b)$, the product $\Pi(a, b)=a b$, the Eukasiewicz t-norm $W(a, b)=\max (a+b-1,0)$ and the drastic t-norm

$$
Z(a, b)=\left\{\begin{array}{cc}
a & \text { if } b=1 \\
b & \text { if } a=1 \\
0 & \text { otherwise }
\end{array}\right.
$$

Their dual t-conorms (with respect to the classical strong negation $N(a)=1-a)$ are, respectively, the maximum $M^{*}(a, b)=\max (a, b)$, the probabilistic sum $\Pi^{*}(a, b)=a+b-a b$, the Eukasiewicz t-conorm or bounded sum $W^{*}(a, b)=\min (a+b, 1)$ and the drastic t-conorm

$$
Z^{*}(a, b)=\left\{\begin{array}{cc}
a & \text { if } b=0 \\
b & \text { if } a=0 \\
1 & \text { otherwise }
\end{array}\right.
$$

Note that, for any t-norm $T$ and t-conorm $S, Z \leqslant$ $T \leqslant M \leqslant M^{*} \leqslant S \leqslant Z^{*}$. 
Proposition $1 A$ continuous t-norm $T$ is Archimedean $(T(a, a)<$ a for all a in $(0,1))$ if and only if it has an additive generator, that is, a strictly decreasing and continuous function $f:[0,1] \rightarrow[0, \infty]$ with $f(1)=0$ such that

$$
T(a, b)=f^{(-1)}(f(a)+f(b)),
$$

where $f^{(-1)}:[0, \infty] \rightarrow[0,1]$ is the pseudo-inverse of $f$, defined by

$$
f^{(-1)}(a)= \begin{cases}f^{-1}(a) & \text { if } a \leqslant f(0), \\ 0 & \text { otherwise. }\end{cases}
$$

An additive generator is defined up to a positive multiplicative constant. On the other hand, if $f$ is an additive generator of a continuous Archimedean t-norm $T$, then $T$ is strict (strictly increasing on $\left.[0,1)^{2}\right)$ if, and only if, $f(0)=\infty$.

The t-norm $\Pi$ is strict with additive generator $f(a)=-\log a$, and the t-norm $W$ is non-strict with additive generator $f(a)=1-a$.

If $T$ is a non-strict continuous Archimedean t-norm with additive generator $f$, then $N(a)=f^{-1}(f(0)-f(a))$ is a strong negation that we call associated to $T$. Note that $T(a, b)=0$ if, and only if, $b \leq N(a)$.

We recall here also the definition of distance.

Definition 3 A function $d: X \times X \rightarrow[0, \infty)$ is a distance on the set $X$ if the following properties are satisfied, for all $a, b, c \in X$ :

1) $d(a, b)=0$ if and only if $a=b$,

2) $d(a, b)=d(b, a)$,

3) $d(a, b) \leqslant d(a, c)+d(c, b)$.

\section{Symmetric difference functions and distances}

Definition 4 A function $\triangle:[0,1] \times[0,1] \longrightarrow[0,1]$ is a symmetric difference function $(S D F)$ if it satisfies for any $a, b \in[0,1]$ :

$\triangle 1) \triangle(a, b)=\triangle(b, a)$,

$\triangle 2) \triangle(a, a)=0, \triangle(a, 0)=a, N(a)=\triangle(a, 1)$ is $a$ strong negation.

Definition 5 Given T, $S, N$ at-norm, a t-conorm, and a strong negation (not necessarily a De Morgan triplet), we define the function:

$$
\triangle(a, b)=S(T(a, N(b)), T(N(a), b))
$$

Next result was mentioned without proof in [2]. For the sake of completeness, we have included it in this paper.

Proposition $2 \triangle$ is a SDF if, and only if, $T(a, N(a))=0 \forall a \in[0,1]$.
In this case we say that $\triangle$ is the SDF associated to the triplet $(T, S, N)$.

Proof If $\triangle$ is a SDF, then $0=\triangle(a, a)=$ $S(T(a, N(a)), T(N(a), a))$ and thus $T(a, N(a))=$ $0 \forall a \in[0,1]$.

Let us suppose now that $T(a, N(a))=0, \forall a \in[0,1]$. Then

$$
\triangle(a, a)=S(T(a, N(a)), T(N(a), a))=S(0,0)=0
$$

On the other hand,

$$
\triangle(a, 0)=S(T(a, N(0)), T(N(a), 0))=S(a, 0)=a
$$

and

$$
\begin{aligned}
\triangle(a, 1) & =S(T(a, N(1)), T(N(a), 1)) \\
& =S(0, N(a))=N(a)
\end{aligned}
$$

Finally,

$$
\begin{aligned}
\triangle(a, b) & =S(T(a, N(b)), T(N(a), b)) \\
& =S(T(N(a), b), T(a, N(b))) \\
& =S(T(b, N(a)), T(N(b), a))=\triangle(b, a)
\end{aligned}
$$

Example 2 The SDF associated to the triplet $\left(W, W^{*}, 1-i d\right)$ is the usual distance on $[0,1]$ : $\triangle(a, b)=|a-b|$.

We are interested in those triplets $(T, S, N)$ such that $\triangle$ defined in (1) is a distance.

Proposition 3 Given a triplet $(T, S, N)$, the function $\triangle$ defined in (1) is a distance if, and only if, the following conditions hold:

i) $T(a, b)=0$ if, and only if, $b \leq N(a)$.

ii) For all $x \in[0,1]$ and any $\epsilon, \delta \in \mathbb{R}$ such that $0 \leq \epsilon \leq 1-x, 0 \leq \delta \leq 1-N(x)$, the following inequality holds (see Figure 1)

$$
T(x+\epsilon, N(x)+\delta) \leq T(x, N(x)+\delta)+T(x+\epsilon, N(x))
$$

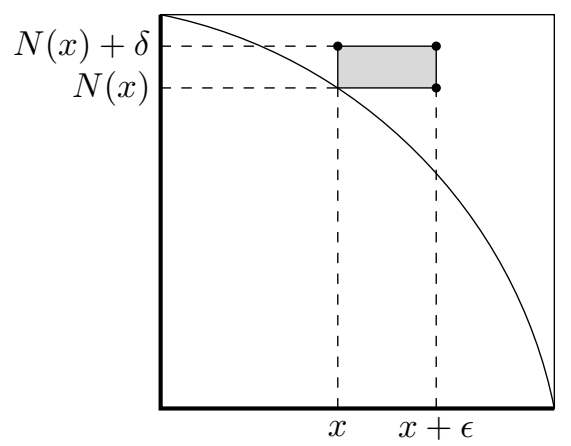

Figure 1: The points involved in the condition (2).

Proof We know that $\triangle$ is symmetric. Let us suppose now that the conditions i) and ii) hold and let us prove that $\triangle$ is a distance. First 
of all, we have from condition i) that $\triangle(a, a)=$ $S(T(a, N(a)), T(N(a), a))=S(0,0)=0$. On the other hand, if $\triangle(a, b)=0$, then $T(a, N(b))=$ $T(N(a), b)=0$ and from condition i), $a \leq b$ and $b \leq a$, thus $a=b$. Now we have to prove the triangular inequality, that is, $\triangle(a, b) \leq \triangle(a, c)+\triangle(c, b)$. From i) we can write

$$
\triangle(a, b)= \begin{cases}T(a, N(b)) & \text { if } b \leq a \\ T(N(a), b) & \text { if } a \leq b\end{cases}
$$

By symmetry, we can suppose that $a<b$; thus we have to consider three cases: $a<b<c, c<a<b$ and $a<c<b$. The triangular inequality for the first two cases follows immediately from the increasingness of $T$. Let us consider now the case $a<c<b$. We have to prove that $T(N(a), b) \leq T(N(a), c)+T(N(c), b)$. This inequality follows from (2) just by taking $x=N(c), \epsilon=N(a)-N(c)$ and $\delta=b-c$.

Conversely, let us suppose now that $\triangle$ is a distance. Since $\triangle(a, a)=0$, we have that $T(a, N(a))=0$ for all $a$, and the monotonicity of $T$ gives that $T(a, b)=0$ if $b \leq N(a)$. Now, if $T(a, b)=0$ for $b>N(a)$, let $c=N(b)$. Thus $\triangle(b, c)=S(T(a, N(c)), T(N(a), c))=S(0,0)=0$, which is impossible since $a>c$. Now we have to prove the condition ii). Let us consider $x \in$ $[0,1], 0<\epsilon \leq 1-x, 0<\delta \leq 1-N(x)$ and $a=N(x+\epsilon), b=N(x)+\delta$ and $c=N(x)$. Thus we have that $a<c<b$ and the triangular inequality gives

$$
\begin{aligned}
T(x+\epsilon, N(x)+\delta) & =T(N(a), b) \\
\leq & T(N(a), c)+T(N(c), b) \\
= & T(x+\epsilon, N(x))+T(x, N(x)+\delta)
\end{aligned}
$$

which is $(2)$.

\section{Remark 1}

i) If we take $\epsilon=1-a, \delta=1-N(a)$ in (3), we have $1 \leq a+N(a)$, that is, $N \geq 1-i d$.

ii) If we take $\delta=1-N(a)$, we have $a+\epsilon \leq$ $a+T(a+\epsilon, N(a))$, that is, $T(a+\epsilon, N(a)) \geq \epsilon$. Analogously, $T(a, N(a)+\delta) \geq \delta$. Thus, if we take $N=1-i d$, we have $T \geq W$.

iii) If $\triangle$ is a distance, then

$$
\triangle(a, b)= \begin{cases}0 & \text { if } a=b \\ T(N(a), b) & \text { if } a<b \\ T(a, N(b)) & \text { if } a>b\end{cases}
$$

Observe that the values of $\triangle$ do not depend on the t-conorm $S$.

iv) Condition (2) can be expressed as a condition of "restricted subadditivity":

$$
T(u \oplus v) \leq T(u)+T(v)
$$

for any $u=w+\vec{\epsilon}, v=w+\vec{\delta}$, where $w=$ $(a, N(a))$ is a vector "on the negation $N ", \vec{\epsilon}=$ $(\epsilon, 0), \vec{\delta}=(0, \delta), 0 \leq \epsilon \leq 1-a, 0 \leq \delta \leq 1-$ $N(a)$, and $u \oplus v=u+v-w$.

Proposition 4 Let $(T, N)$ satisfying the conditions in Proposition 3. If $T$ is continuous on the graph of $N(\{(x, N(x)) ; x \in[0,1]\})$, then it is continuous on all its domain.

Proof Let us suppose that $T$ is discontinuous at $(a, b)$ with $b>N(a)$. Thus either $T(\cdot, b)$ is discontinuous at $a$ or $T(a, \cdot)$ is discontinuous at $b$. Let us suppose first that $T(\cdot, b)$ is right-discontinuous at $a$. Then there exists $\lambda>0$ such that

$$
T(a+\epsilon, b)-T(a, b) \geq \lambda \forall \epsilon>0
$$

From condition (2) we have $T(a+\epsilon) \leq T(a, b)+$ $T(a+\epsilon, N(a))$, that is

$$
\begin{aligned}
\lambda & \leq T(a+\epsilon, b)-T(a, b) \\
& \leq T(a+\epsilon, N(a)) \\
& =T(a+\epsilon, N(a))-T(a, N(a))
\end{aligned}
$$

for all $\epsilon>0$, and thus $T$ is not right-continuous at $(a, N(a))$.

Let us suppose now that $T(\cdot, b)$ is leftdiscontinuous at $a$. Then there exists $\lambda>0$ such that

$$
\forall \epsilon>0, T(a, b)-T(a-\epsilon, b) \geq \lambda
$$

From condition $(2)$ we have $T(a, b) \leq T(a-\epsilon, b)+$ $T(a, N(a-\epsilon))$, that is

$$
\begin{aligned}
\lambda & \leq T(a, b)-T(a-\epsilon, b) \\
& \leq T(a, N(a-\epsilon)) \\
& =T(a, N(a-\epsilon))-T(a, N(a))
\end{aligned}
$$

for all $\epsilon>0$. Then, from the continuity of $N$, we have

$$
\lambda \leq T(a, N(a)+\epsilon))-T(a, N(a))
$$

for all $\epsilon>0$, and thus $T$ is not right-continuous at $(a, N(a))$.

The proof for the case of $T(a, \cdot)$ is completely analogous and it has been omitted.

Proposition 5 Given a triplet $(T, S, N)$ with $T$ a continuous t-norm, the function $\triangle$ defined in (1) is a distance if, and only if, the following conditions hold:

i) $T$ is a non-strict archimedean t-norm with associated negation $N$.

ii) The function $f^{-1}(1-i d)$ is subadditive, where $f$ is the normalized additive generator of $T$ $(f(0)=1)$. 
Proof If $\triangle$ is a distance, then the condition i) of Proposition 3 proves that $T$ is a non-strict archimedean t-norm with associated negation $N$. Let now $f$ be the additive generator of $T$ with $f(0)=1$. Thus $N(a)=f^{-1}(1-f(a))$ and the expression of $\triangle$ becomes

$$
\triangle(a, b)= \begin{cases}0 & \text { if } a=b \\ f^{-1}(1-f(a)+f(b)) & \text { if } a<b \\ f^{-1}(1+f(a)-f(b)) & \text { if } a>b\end{cases}
$$

that is, $\triangle(a, b)=f^{-1}(1-|f(a)-f(b)|)$. Now the triangular inequality for the case $a<c<b$ becomes $f^{-1}(1-(f(a)-f(b))) \leq f^{-1}(1-(f(a)-f(c)))+$ $f^{-1}(1-(f(c)-f(b)))$. If we take $u=f(a)-f(b)$ and $v=f(c)-f(b)$, we obtain

$$
f^{-1}(1-(u+v)) \leq f^{-1}(1-u)+f^{-1}(1-v)
$$

for all $u, v \geq 0$ such that $u+v \leq 1$. Thus $f^{-1}(1-i d)$ is subadditive.

Conversely, let us suppose now that the conditions i) and ii) hold. From previous results, we only have to prove the triangular inequality of $\triangle$. But this result comes immediately from the above reasoning.

\section{Remark 2}

i) Note that $(T, S, N)$ does not need to be a De Morgan triplet.

ii) The function $f^{-1}(1-i d)$ is subadditive if, and only if, $1-f$ is superadditive.

iii) If the t-norm $T$ is a copula and $\triangle$ is a distance, then $f$ is convex and thus $f^{-1}(1-i d)$ is superadditive. Then $f^{-1}(1-i d)$ is additive, thus $f^{-1}(1-i d)=i d$ and therefore $f=1-i d$, that is $T=W$.

iv) Let us observe that the if $f^{-1}(1-i d)$ is subadditive then $N \geq 1-i d$.

Proposition 6 If the additive generator $f$ of $T$ is concave, then the condition ii) of Proposition 5 holds. The converse is not true, in general.

Proof If $f$ is concave, then $f^{-1}$ and $h=f^{-1}(1-i d)$ are also concave. Since $h(0)=0$, the function $h$ is subadditive. To prove that the converse is not true, let us consider $f=g^{-1}$, where $g(a)=-a^{3}+a^{2}-$ $a+1$. The function $f$ is not concave (since $g$ is not concave), but $f^{-1}(1-i d)=g(1-i d)$ is subadditive. Thus the concavity of $f$ is not a necessary condition for (2) to hold.

Example 3 The generator of the Yager t-norms, $f(a)=(1-a)^{\lambda}$ where $0 \leq \lambda \leq 1$, is a concave function. The associated distance is

$$
\begin{aligned}
\triangle(a, b) & =f^{-1}(1-|f(a)-f(b)|) \\
& =1-\left(1-\left|(1-a)^{\lambda}-(1-b)^{\lambda}\right|\right)^{1 / \lambda}
\end{aligned}
$$

The generator of the Sugeno-Weber t-norms, $t_{\lambda}(a)=1-\frac{\ln (1+\lambda a)}{\ln (1+\lambda)}$, is a concave function for any $\lambda \in(-1,0)$. The associated distance is

$$
\begin{aligned}
\triangle(a, b) & =f^{-1}(1-|f(a)-f(b)|) \\
& =\frac{1+\lambda}{\lambda} \exp \left\{\frac{1}{\ln (1+\lambda)} \cdot\left|\ln \frac{1+\lambda b}{1+\lambda a}\right|\right\}
\end{aligned}
$$

Proposition 7 Let us consider a triplet $(T, S, N)$ such that $T(a, b)=0$ if, and only if, $b \leq N(a)$. If $N$ is concave and $T$ is concave in each variable on its positive region, then the condition (2) holds.

Proof Let $a \in[0,1]$, and $\epsilon, \delta \in \mathbb{R}$ such that $0 \leq \epsilon \leq 1-a, 0 \leq \delta \leq 1-N(a)$. If we take $\alpha=\frac{N(a)-N(a+\epsilon)}{N(a)+\delta-N(a+\epsilon)}$, then we can write $(a+\epsilon, N(a))=\alpha \cdot(a+\epsilon, N(a)+\delta)+(1-\alpha) \cdot(a+$ $\epsilon, N(a+\epsilon))$.

Analogously, we have $(a, N(a)+\delta)=$ $\alpha^{\prime} \cdot(a+\epsilon, N(a)+\delta)+\left(1-\alpha^{\prime}\right) \cdot(N(N(a)+\delta), N(a)+\delta)$, where $\alpha^{\prime}=\frac{a-N(N(a)+\delta)}{a+\epsilon-N(N(a)+\delta)}$.

Since $T$ is concave and it equals 0 on the negation $N$, we have $T(a+\epsilon, N(a)) \geq \alpha \cdot T(a+\epsilon, N(a)+\delta)$ and $T(a, N(a)+\delta) \geq \alpha^{\prime} \cdot T(a+\epsilon, N(a)+\delta)$. By adding this two inequalities, we obtain $T(a+\epsilon, N(a))+$ $T(a, N(a)+\delta) \geq\left(\alpha+\alpha^{\prime}\right) \cdot T(a+\epsilon, N(a)+\delta)$. Thus, if we prove that $\alpha+\alpha^{\prime} \geq 1$, we will have the condition (2). Now a straightforward calculation proves that $\alpha+\alpha^{\prime} \geq 1$ is equivalent to

$$
(a-N(N(a)+\delta)) \cdot(N(a)-N(a+\epsilon)) \geq \epsilon \cdot \delta
$$

and this inequality holds since $N$ is concave (see Figure 2).

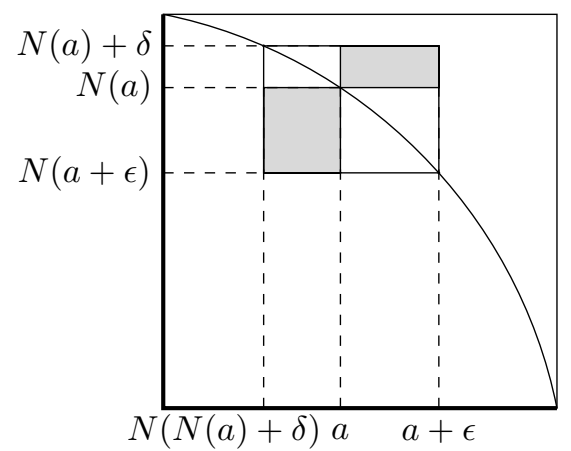

Figure 2: The points involved in the proof of Proposition 7 .

Remark 3 Under the conditions above, condition (2) plus $T$ concave in each variable in its positive region do not imply that $N$ is concave. Moreover, condition (2) plus $N$ concave do not imply that $T$ is concave in each variable in its positive region. Let see two examples. 
Example 4 Let $N$ be a strong negation. Let us consider the (left-continuous but not continuous) $t$ norm $M_{N}$ given by

$$
M_{N}(a, b)= \begin{cases}0 & \text { if } b \leq N(a) \\ \min (a, b) & \text { if } b>N(a)\end{cases}
$$

It can be proved that for any t-conorm $S$, $\left(M_{N}, S, N\right)$ defines a distance through (1) if, and only if, $N \geq 1-i d$. This distance is given by

$$
\triangle(a, b)= \begin{cases}0 & \text { if } a=b \\ \min (N(a), b) & \text { if } a<b \\ \min (a, N(b)) & \text { if } a>b\end{cases}
$$

In the case when $N=1-i d\left(M_{1-i d}=T^{n M}\right.$, the nilpotent minimum), this distance becomes

$$
\triangle(a, b)= \begin{cases}0 & \text { if } a=b \\ b & \text { if } a<b, a+b \leq 1 \\ 1-a & \text { if } a<b, a+b \geq 1 \\ a & \text { if } a>b, a+b \leq 1 \\ 1-b & \text { if } a>b, a+b \geq 1\end{cases}
$$

(see Figure 3).

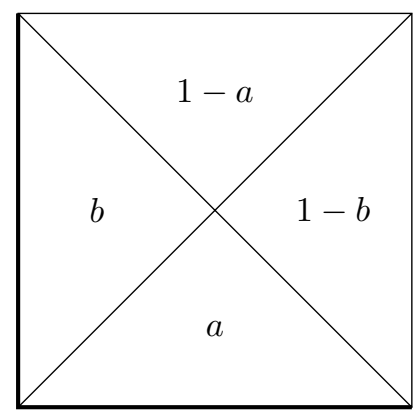

Figure 3: Structure of the distance $\triangle(a, b)$ in Example 4 , for the case $N=1-i d$.

The following result can be found in [6].

Proposition 8 Given a strong negation $N$, the $t$ norms $T$ such that

1) $T(a, b)=0$ when $b \leq N(a)$

2) $T$ is positive and continuous in the region $\{(a, b): b>N(a)\}$

have the form

$$
T(a, b)=\left\{\begin{array}{l}
0, \\
\text { if } b \leq N(a) \\
\alpha+(\beta-\alpha) T_{1}\left(\frac{a-\alpha}{\beta-\alpha}, \frac{b-\beta}{\beta-\alpha}\right) \\
\text { if } b>N(a), \max (a, b)<\beta(\alpha \neq \beta) \\
\min (a, b), \\
\text { if } b>N(a), \max (a, b) \geq \beta
\end{array}\right.
$$

where $0 \leq \alpha \leq \beta \leq 1, N(\alpha)=\beta$, and $T_{1}$ is a continuous and non-strict archimedean $t$-norm with zero region $\left\{(a, b): b \leq N_{\alpha}^{\beta}(a)\right\}$, where $N_{\alpha}^{\beta}$ is the strong negation defined by $N_{\alpha}^{\beta}(a)=\frac{N((\beta-\alpha) a+\alpha)-\alpha}{\beta-\alpha}$ (see Figure 4).

\section{Remark 4}

i) If $\alpha=0$ and $\beta=1$, then $N_{0}^{1}=N$, and $T$ is a continuous and non-strict archimedean t-norm with zero region $\{(a, b): b \leq N(a)\}$.

ii) The case $\alpha=\beta$ (point of symmetry of the negation $N)$ means that $T$ has the form:

$$
T(a, b)= \begin{cases}0 & \text { if } b \leq N(a) \\ \min (a, b) & \text { if } b>N(a) \\ & \max (a, b) \geq \beta\end{cases}
$$

that is, $T=M_{N}$.

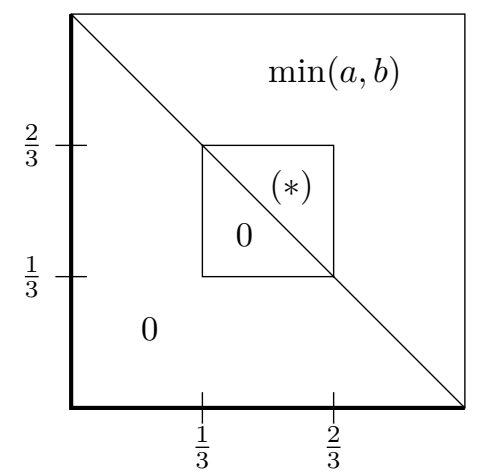

Figure 4: The structure of the t-norm in Proposition 8 for $\alpha=1 / 3, \beta=2 / 3$ and $N=1-i d$, where $(*)$ stands for $\alpha+(\beta-\alpha) T_{1}\left(\frac{a-\alpha}{\beta-\alpha}, \frac{b-\beta}{\beta-\alpha}\right)$.

According to Proposition 3, Proposition 5, and Example 4, we have

Proposition 9 For the t-norms $T$ of the form given in (5), the function $\triangle$ defined in (1) is a distance if, and only if, the following conditions hold:

i) $N \geq 1-i d$.

ii) $1-f$ is superadditive, where $f$ is the normalized additive generator of the t-norm $T_{1}$ (with $\alpha \neq$ $\beta)$.

\section{Conclusions}

We present a full description of those triplets $(T, S, N), T$ a t-norm, $S$ a t-conorm and $N$ a strong negation, such that the symmetric difference function $\triangle(a, b)=S(T(a, N(b)), T(b, N(a))$ is a distance.

\section{Acknowledgements}

The authors acknowledge the support of the Spanish DGI grants TIN2013-42795-P, TIN2014-56381REDT and Programa pont La Caixa d'ajut a grups de recerca (2014). 


\section{References}

[1] I. Aguiló, J. Martín, G. Mayor, J. Suñer: On distances derived from t-norms. Fuzzy Sets and Systems, In press, 2014, doi:10.1016/j.fss.2014.09.021

[2] I. Aguiló, T. Calvo, J. Martín, G. Mayor, J. Suñer: Distancias y Multidistancias Derivadas de Operadores de Diferencia Simétrica. Proc. of Estylf 2014, pp. 309-314, 2014.

[3] C. Alsina: On some metrics induced by copulas. In: Walter (Ed.), General Inequalities 4, p. 397, 1984.

[4] C. Alsina, E. Trillas: On the symmetric difference of fuzzy sets. Fuzzy Sets and Systems 153, pp. 181-194, 2005.

[5] C. Alsina, M.J. Frank, B. Schweizer: Associative Functions: Triangular Norms and Copulas. World Scientific Publishing Company, Singapur, 2006.

[6] S. Jenei: New family of triangular norms via contrapositive symmetrization of residuated implications. Fuzzy Sets and Systems 110, pp. 157-174, 2000.

[7] E. P. Klement, R. Mesiar, E. Pap: Triangular Norms. In: "Trends in Logic - Studi Logica Library", 8. Kluwer Academic Publishers, 2000. 